\title{
Long-term follow-up of joint stabilization procedures in the treatment of fixed deformities of feet in leprosy
}

\author{
M. EBENEZER, S. PARTHEEBARAJAN \\ \& S. SOLOMON \\ Branch of Surgery and Surgical Rehabilitation Schieffelin Leprosy \\ Research and Training Centre Karigiri $632106 \mathrm{NA}$ A Dt \\ Tamilnadu, India
}

Accepted for publication 24 October 1995

\begin{abstract}
Summary This retrospective study of 52 patients, who underwent joint stabilization procedures for static deformities of the feet in leprosy between 1971 and 1985 , was undertaken to assess the long-term results of joint stabilization of feet for fixed deformities in leprosy. The main purpose of joint stabilization is to make the feet plantigrade for weight bearing and to make the wearing of footwear possible. Deformities corrected include varus, equinus and equinovarus. Chronic ulceration occurs repeatedly if these deformities are not corrected and leads to inevitable bone destruction and eventual amputation.
\end{abstract}

\section{Introduction}

Fixed deformities involving the ankle and subtalar joints are common in leprosy. They occur as a result of infection, trauma, neuroarthropathy and tissue destruction each contributing to the final position of the foot. In these deformities the plantar skin is deflected away from the line of weight-bearing resulting in areas that normally do not bear weight to lie along the line of weight bearing. ${ }^{1}$ Lateral border of the foot in the case of a varus deformity and medial border of the foot in valgus deformity bear weight during walking and are subject to abnormal pressures. In deformities like equinus and rocker bottom foot the forefoot and the midfoot are subject to high pressures during walking. Because of these abnormal pressures chronic ulceration is common at these sites. ${ }^{2}$

Left alone these deformities follow a natural course of repeated chronic ulceration leading to inevitable bone destruction and eventual amputation. ${ }^{3,4}$ Hence these def ormities necessitate the performing of various joint stabilization procedures to correct deformity, relieve abnormal pressures at these sites and to restore good plantar skin which is deflected away for weight bearing.

Brand recognized the role of triple arthrodesis in correcting some deformities of the good. ${ }^{5}$ In 1971 Fritschi discussed the place of arthrodesis in severely deformed feet. ${ }^{6}$ Shibata et al. reported $73 \%$ fusion rate in deformities of leprotic neuropathic feet. ${ }^{7}$ This 


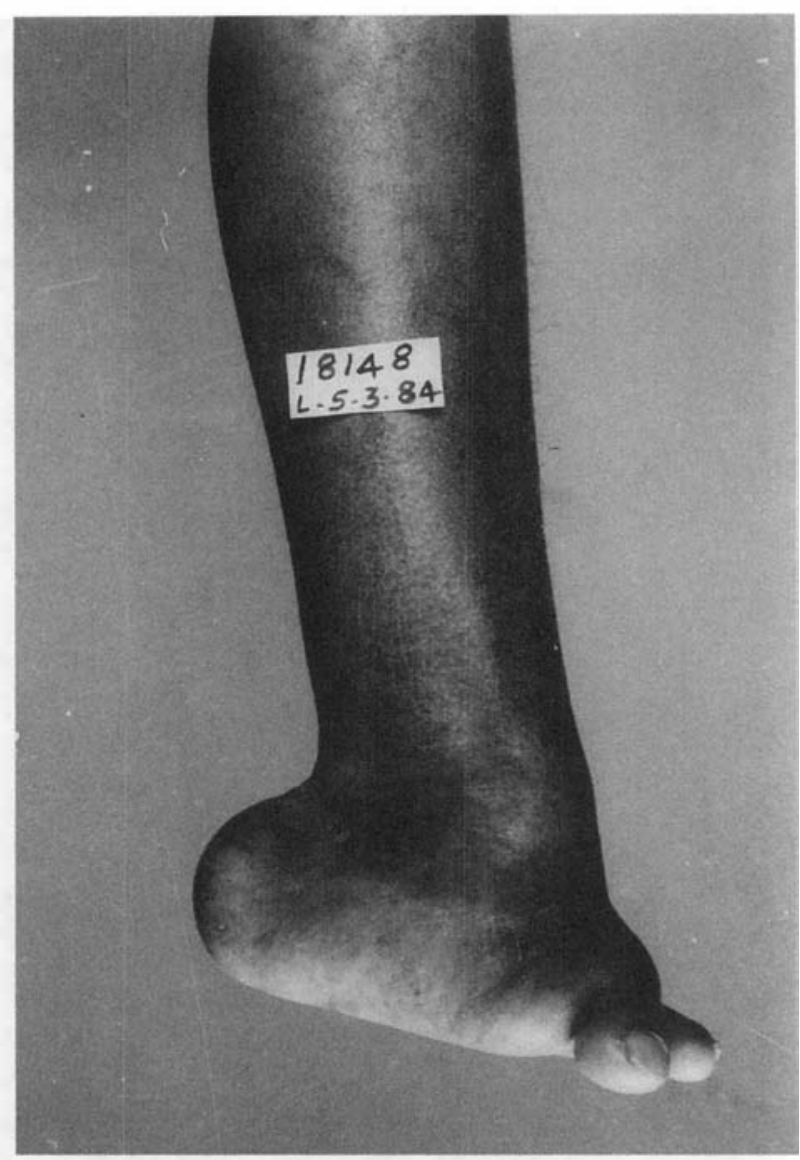

Figure 1. Equinus component of an equinovarus deformity.

retrospective study was undertaken to assess the long-term results of joint stabilization of feet for fixed deformities in leprosy.

\section{Methods}

Patients who underwent joint stabilization procedures for static deformities of the feet at the Schieffelin Leprosy Research and Training Centre, Karigiri provide the material for this study.

The main purpose of joint stabilization procedure is to correct the deformity to make the foot plantigrade for weight bearing and to make the foot fit for footwear usage. The type of foot deformities corrected include varus, equinus and equinovarus. (Figures 1-2.)

The following criteria were used in selecting the patient for a joint stabilization procedure: both feet should be ulcer free for at least 3 weeks; the available weightbearing surface should be more than one third of the normal; there should be reasonable bone stock though there may be disorganization; patients should be willing for six 


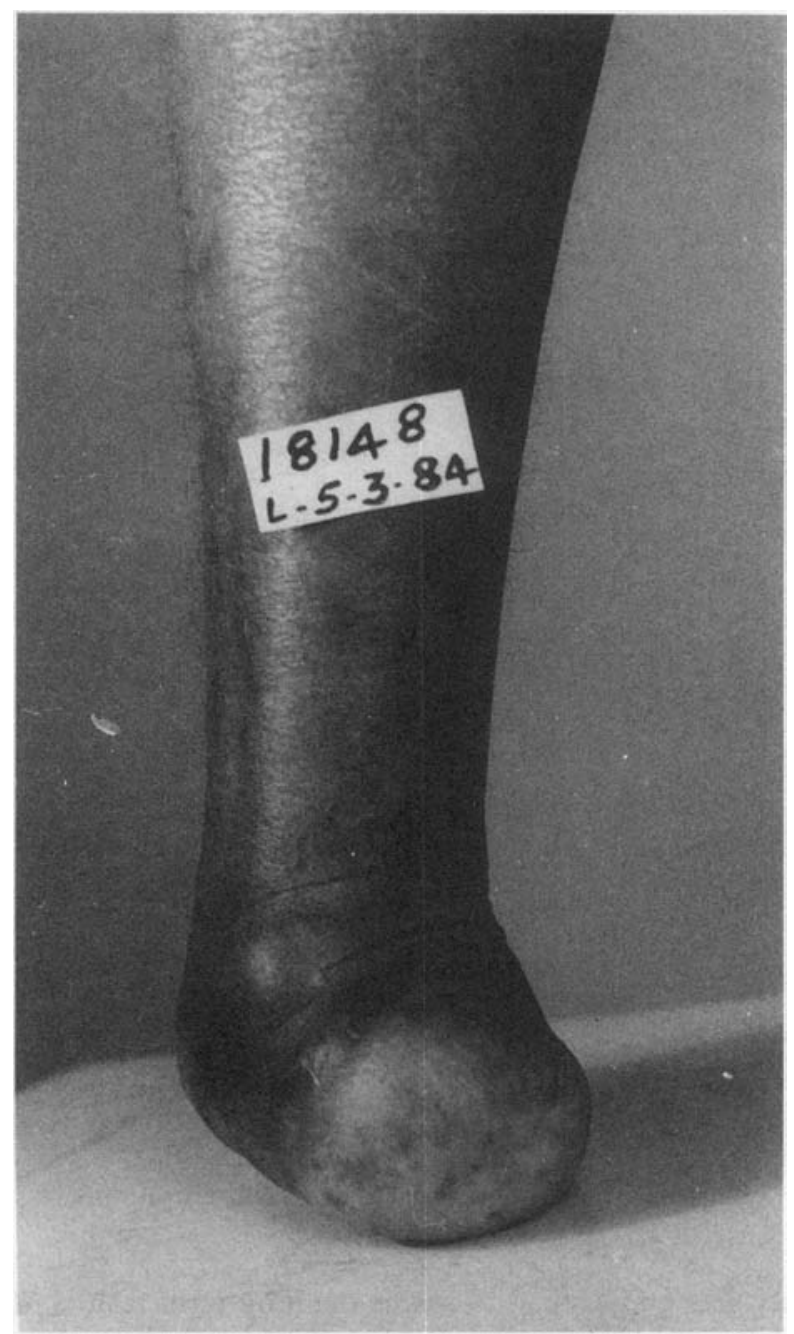

Figure 2. Varus component of an equinovarus deformity.

months of immobilization in a cast followed by the wearing of specialized footwear (which are heavy and costly) for at least one year.

Surgery consisted of adequate exposure of the concerned joints and removal of adequate wedges from the adjacent joint surfaces to obtain correction of the deformity. The corrected position was maintained by means of staples or compression clamps.

Following surgery the foot was immobilized in a nonweight-bearing cast for 1 month. This was followed by weight-bearing casts for the next 5 months at which time the union was checked clinically and radiologically. If the union was satisfactory the patient was provided with a fixed ankle brace to protect the union till consolidation of the union occurred. 


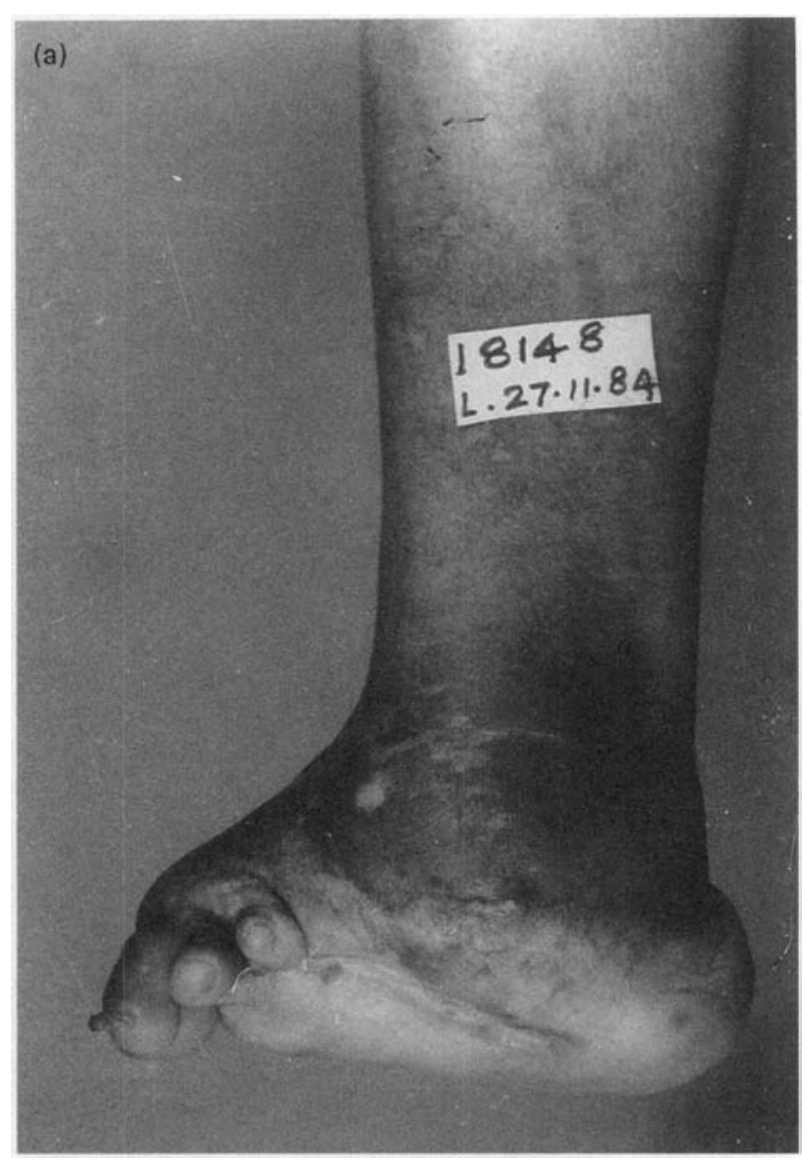

Figure 3(a).

At each follow-up visit the position of the foot, clinical union and ulcer episodes over the follow-up period were noted. These patients were followed up initially at intervals of six months for the first two years and thereafter whenever the patient had a problem with his foot or footwear.

\section{Patients}

Fifty-two patients underwent joint stabilization procedures for static deformities of feet in leprosy between 1971 and 1985 at the Schieffelin Leprosy Research and Training Centre.

AGE, SEX AND CLASSIFICATION

The details of age, sex and classification are given in Table 1. Most patients were between 


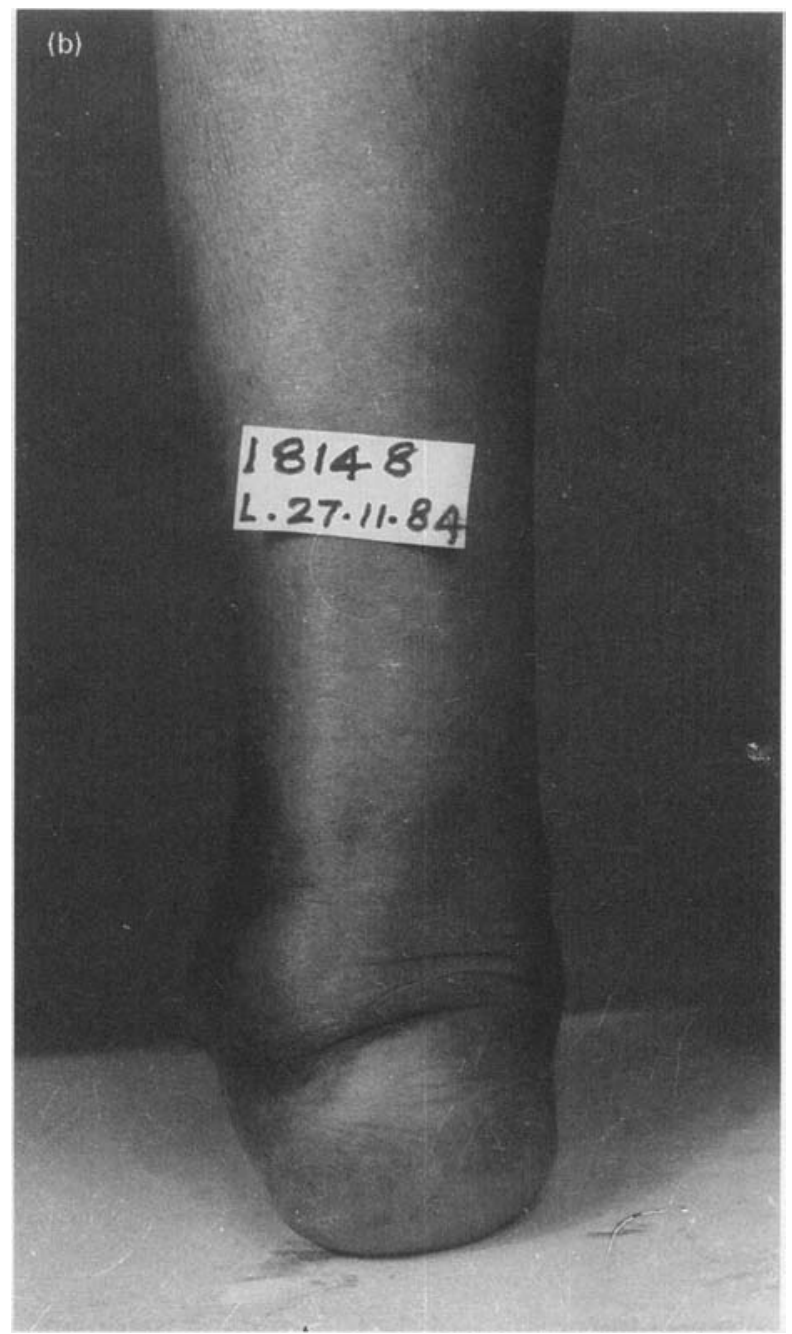

Figure 3. Correction of both equinus (a) and varus (b).

Table 1. Age and sex distribution of patients

\begin{tabular}{lrcr}
\hline Age (years) & Male & Female & Total \\
\hline $21-30$ & - & 2 & 2 \\
$31-40$ & 7 & 6 & 13 \\
$41-50$ & 18 & 4 & 22 \\
$51-60$ & 10 & 3 & 13 \\
$61-70$ & 2 & - & 2 \\
Total & 37 & 15 & 52 \\
\hline
\end{tabular}


Table 2. Deformity and site of ulcer

\begin{tabular}{lcccc}
\hline Site of ulcer & Varus & Equinus & Equinovarus & Total \\
\hline Lateral border & 42 & 1 & 2 & 45 \\
Foref oot & 1 & 8 & 2 & 11 \\
Total & 43 & 9 & 4 & 56 \\
\hline
\end{tabular}

31 and 60 years of age. Thirty-six men and 16 women underwent joint stabilization of the feet. Thirty-four of them belonged to the tuberculoid type and 18 to the lepromatous type. Four patients underwent joint stabilization on both sides making the total number of feet operations, 56.

TYPE OF DEFORMITY AND TYPE OF SURGERY

Type of deformity, details of the ulcer and the type of surgery performed are shown in Table 2. Varus deformity was seen in 43 feet, equinus in 9 cases and equinovarus in 4 cases. There was chronic ulceration or scarring in 45 patients over the lateral border of the foot and in 11 patients in the forefoot. The chronicity of the ulcer varied from 2 years to 20 years.

Subtalar arthrodesis was done in 42 patients, ankle arthrodesis in 10 and pantalar arthrodesis in 4 cases (Table 3). Internal fixation with staples was done in 30 feet and with compression clamps in 18 cases. Eight feet did not have any internal fixation.

\section{FOOTWEAR AND FOOT PRINTS}

Fifty-three feet were prescribed a fixed ankle brace and 3 a patellar tendon-bearing brace. Harris mat foot prints were done to check that the distribution of pressure at the time of fitting the footwear was satisfactory in all cases.

\section{FOLLOW UP}

Eleven patients were lost to follow up. The profile of patients lost to follow u in terms of age, sex, classification, type of deformity and type of surgery is very similar to the entire group. Two patients had below the knee amputations because of underlying bacterial

Table 3. Deformity and type of surgery

\begin{tabular}{lcccr}
\hline Type of surgery & Varus & Equinus & Equinovarus & Total \\
\hline Triple arthrodesis & 42 & - & - & 42 \\
Ankle arthrodesis & - & 10 & - & 10 \\
Pantalar arthrodesis & & - & 4 & 56 \\
Total & 42 & 10 & & \\
\hline
\end{tabular}


Table 4. Results at follow up

\begin{tabular}{lrr}
\hline & No & $\%$ \\
\hline $\begin{array}{lr}\text { Position of the foot } \\
\text { Neutral position }\end{array}$ & 31 & $72 \cdot 1$ \\
$<10$ degrees def ormity & 9 & $20 \cdot 9$ \\
10-15 degrees def ormity & 3 & $7 \cdot 0$ \\
State of union & & \\
Bony union & 43 & $100 \cdot 0$ \\
Radiological union & 43 & $100 \cdot 0$ \\
Ulcer rate & & \\
(Av. ulcer episodes per year) & & \\
$0 \cdot 00$ & 6 & $14 \cdot 0$ \\
$0 \cdot 10-0 \cdot 50$ & 25 & $58 \cdot 1$ \\
$0 \cdot 60-0 \cdot 90$ & 10 & $23 \cdot 3$ \\
$1 \cdot 00$ or more & 2 & $4 \cdot 6$ \\
& & \\
\hline
\end{tabular}

infection. Thus, 39 patients with 43 joint stabilizations left in the study were followed up for an average of 11.93 years (5-24 years). Only patients who were followed up for a minimum of 5 years were included in the analysis of results.

\section{Results}

The follow up results are shown in Table 4.

POSITION OF THE FOOT

The position of the foot at follow up was neutral in 31 patients. There was a residual def ormity of less than $10^{\circ}$ in 9 cases and $10-15^{\circ}$ in 3 cases.

STATE OF UNION

At the six-month follow-up visit all feet had sound clinical and radiological union.

\section{ULCER RATE AFTER CORRECTION}

The incidence of plantar ulceration during the follow-up period was 0.43 per year per person or $36 \cdot 1$ per 1000 person months.

\section{COMPLICATIONS}

As mentioned earlier two patients needed below the knee amputation due to severe bacterial infection. Pin tract infection was seen in three cases which settled down after the removal of pins. Revision of arthrodesis was required in 4 cases. Revision was 
required in 3 cases due to recurrence of the deformity, at 2 years, 3 years and 11 years and in one case after 2 months because of incomplete correction.

\section{Discussion}

Fixed deformities of the feet having good weight-bearing plantar surface, little scarring and well-preserved bony architecture can be corrected by joint stabilization and present no difficulty in making such a decision. It is when one is faced with a fixed deformity of a foot having reduced plantar surface with gross scarring and disorganized bony architecture that making a decision to salvage the foot as against an amputation becomes difficult to make.

Fritschi felt that correcting and preserving such a limb despite the cost in terms of loss of man hours and restriction of activities of the patient would be worthwhile because it is always preferable for the patient to have a foot of his own which in an emergency he can immediately use without having to find one and buckle it on. ${ }^{6}$ The usefulness of this conservatism needed to be clearly proved in the long term since the salvaged anaesthetic foot continues to be at risk of developing ulceration with the possibility of further destruction and the inevitable amputation.

This study demonstrates that joint stabilization has enabled the patient to have the benefits of the use of his own limb on average for more than a decade after surgical correction. The benefits of the surgery were a plantigrade foot for weight bearing and the fitting of footwear. The patient was also relatively f ree from chronic ulceration of the feet making them more acceptable at home and work.

It has been brought out in the study that even after correction of deformity and appropriate footwear usage the patient is still prone to plantar ulceration since the basic impairment, namely anaesthesia and motor paralysis of the foot has not changed.

Subtalar arthrodesis for correcting a varus deformity and ankle arthrodesis for correcting an equinus deformity are the methods of stabilization recommended. In an equinovarus deformity where both equinus and varus need to be corrected a singlestaged pantalar arthrodesis is useful. However, the decision of procedure would depend on the type of deformity and the pattern of bony configuration seen radiologically.

The bony configuration in deformed anaesthetic feet is often distorted and it is difficult to identify normal anatomical landmarks. Because of repeated ulceration, sequestration and neuroarthropathy there is bone loss and the bone stock is of ten insufficient. The bones of the foot are very soft due to gross osteoporosis making accurate wedge taking difficult and to get an internal fixation in place. Neuroarthropathy was seen only in two cases in this series and in general may not be the main contributing factor in the development of fixed deformities of the feet.

Since most patients $(94 \%)$ ) were in their fourth to six th decades of life, preservation of the patient's own limb helped the patient to contribute positively to the society during the most productive years of his life.

In conclusion correction of static deformities of the feet in leprosy by joint stabilization procedures helps the patient to retain his own limb with all its advantages. 


\section{Acknowledgment}

The authors would like to acknowledge the help of Mr P. Andrews, Co-ordinator Surgical Department for collection and compiling of data.

\section{References}

${ }^{1}$ Fritschi EP. Surgical Reconstruction and Rehabilitation in Leprosy, 1984, pp 210-220. The Leprosy Mission, New Delhi.

${ }^{2}$ Clawson DK, Seddon HJ. The late consequences of sciatic nerve injury. J Bone Joint Surg, 1960, 42-B: 213.

${ }^{3}$ Lennox W M. The surgical management of foot def ormities. Lepr Rev, 1965; 36: $27-34$.

${ }^{4}$ Lennox WM. A classification of leprosy foot deformities. Lepr Rev, 1964; 35: 245-9.

${ }^{5}$ Hodges WAA. Treatment of foot deformities in leprosy. E. Africa Med J, 1956; 33: 301-3.

${ }^{6}$ Fritschi EP. Deformed feet in leprosy. Int J Lepr, 1971; 39: 621-5.

${ }^{7}$ Shibata T, Tada K, Kagawa, Hshizume C. The results of arthrodesis of the ankle for leprotic neuroarthropathy. J Bone Joint Surg, 1990; 72(5)-A: 749-56. 